\title{
Ex Vivo Development of Eggs from Head Lice (Pediculus humanus capitis)
}

\author{
Susanne Sonnberg ${ }^{1}$, Fabíola A. Oliveira ${ }^{2,3}$, Iana L. Araújo de Melo ${ }^{4}$, Matheus M. de Melo Soares ${ }^{4}$, \\ Heiko Becher ${ }^{1}$ and Jorg Heukelbach ${ }^{*}, 2,4$
}

\author{
${ }^{I}$ Institute of Public Health, University of Heidelberg, Heidelberg, Germany \\ ${ }^{2}$ Anton Breinl Centre for Public Health and Tropical Medicine; School of Public Health, Tropical Medicine and \\ Rehabilitation Sciences, James Cook University, Townsville, Australia \\ ${ }^{3}$ Health Department of the City of Cologne, Counselling Center for Sexually Transmitted Diseases/AIDS, Cologne, \\ Germany \\ ${ }^{4}$ Department of Community Health, School of Medicine, Federal University of Ceará, Fortaleza, Brazil
}

\begin{abstract}
Background: Data on ovicidal activity of pediculicides are essential to determine the efficacy of commercial products, and to define application schemes. We present an adaptation of historical methods for rearing head lice, and describe their development ex vivo.

Methods: Adult head lice were collected and fed on volunteers to obtain fertile eggs of known age. Two methods of feeding were applied: interval feeding (performed every 8-12 hours), and continuous feeding (placing lice in a round Plexiglas receptacle, closed with gauze attached to the skin). Eggs were incubated at $25-28,6^{\circ} \mathrm{C}$ and $51,8 \%-69,3 \%$ relative humidity. The following outcome measures were used: appearance of eye spot; presence of embryonic structures; presence of embryonic movements; and hatching. A total of $340 \mathrm{eggs}$ were obtained by continuous feeding. Of these, 205 were examined daily. The remaining 135 eggs and those obtained by interval feeding $(n=159)$ were observed once, to confirm hatching status.

Results: Eye spots of the embryos started to appear 6 days after oviposition (median $=7$ days), and embryonic movements were first seen 9 days after oviposition (median $=11$ days). Interval feeding of head lice revealed a hatch rate of $10,7 \%$ $(17 / 159)$, as compared to a hatch rate of $80,9 \%(275 / 340)$ achieved with continuous feeding. Hatching started 9 days after oviposition (median $=12$ days). Skin irritation (papular rash; moderate to severe itching) appeared after continuous feeding of adult lice on volunteers.

Conclusions: Continuous feeding of head lice on a human host is a suitable approach to obtain head lice eggs for ovicidal testing. The method is simple and cheap, offers conditions similar to the natural habitat, and hatch rates are high. However, side effects on volunteers are considerable.
\end{abstract}

Keywords: Embryonal development, head lice, life cycle, pediculosis.

\section{INTRODUCTION}

Increasing prevalence of head lice infestation (pediculosis) is a worldwide public health concern. Pediculosis occurs primarily in school-aged children, but may affect all age groups and socioeconomic levels [1,2]. The recent international increase in the prevalence of pediculosis has been suggested to result from incorrect use of topical insecticides, and consequently growing resistance to commonly used pediculicides with a neurotoxic mode of action, such as those containing permethrin and malathion [3-7].

In addition to information on adulticidal efficacy, data on ovicidal activity are essential to determine the efficacy of a certain product and to define application schemes of

*Address correspondence to this author at the Departamento de Saúde Comunitária, Faculdade de Medicina, Universidade Federal do Ceará, Rua Prof. Costa Mendes 1608, 5. Andar, Fortaleza CE 60430-140, Brazil; Tel: ++55-85-33668045; Fax: ++55-85-33668050; E-mail: heukelbach@web.de topical compounds [8]. For example, considering the low ovicidal efficacy of many compounds, product leaflets usually recommend a second topical application after 7-10 days. Repeating the treatment after this period ensures that nymphs and adult lice come in contact with the product after hatching before they mature, mate and lay eggs. The situation in practice is further complicated, as many producers of anti-lice products recommend adjunctive combing and sometimes provide a free lice comb with the product. In addition, commercialized products are not bound by health authorities to meet a minimum level of efficacy [8]. Consequently, health professionals and consumers often cannot determine if effectiveness is due to adulticidal and ovicidal properties of the product, or a result of mechanical removal of adults and eggs by adjunctive combing. Thus, there is a clear need for adequately designed and standardized adulticidal and ovicidal ex vivo assays [8-10].

Head lice eggs for bioassays can be collected from infested individuals, but their age will not be exactly known, and rearing conditions would not be standardized. Eggs of 
known age and reared under standardized conditions can be obtained by in vivo or in vitro rearing of lice. The latter is achieved by an automated apparatus that allows rearing of head lice through the entire life cycle, with minimal human maintenance [11, 12]. An effective animal model for rearing head lice, as it has been used for body lice, could not yet be established [13].

Reliable data on ovicidal efficacy requires testing of fertile eggs with high hatch rates at different stages of development, as susceptibility to insecticides may vary according to the development of the embryo [9]. This can be achieved in an environment that closely mimics the natural habitat of head lice, which means that head lice would feed on human hosts, either by intermittent or continuous feeding. Nuttall (1917) [14] reared head lice on human hosts by feeding adult lice continuously, using a wristlet (Fig. 1). About 60 years later, Lang and Roan (1974) [15] modified this method by using a watchstrap with a plastic container (Fig. 2). Both authors used a small device tampered by chiffon or nylon mesh. The device was positioned on the skin of volunteers and allowed lice to feed ad libitum. However, these and other historical publications report that skin irritations and itching occurred, caused by saliva injected by the lice to facilitate feeding $[16,17]$.

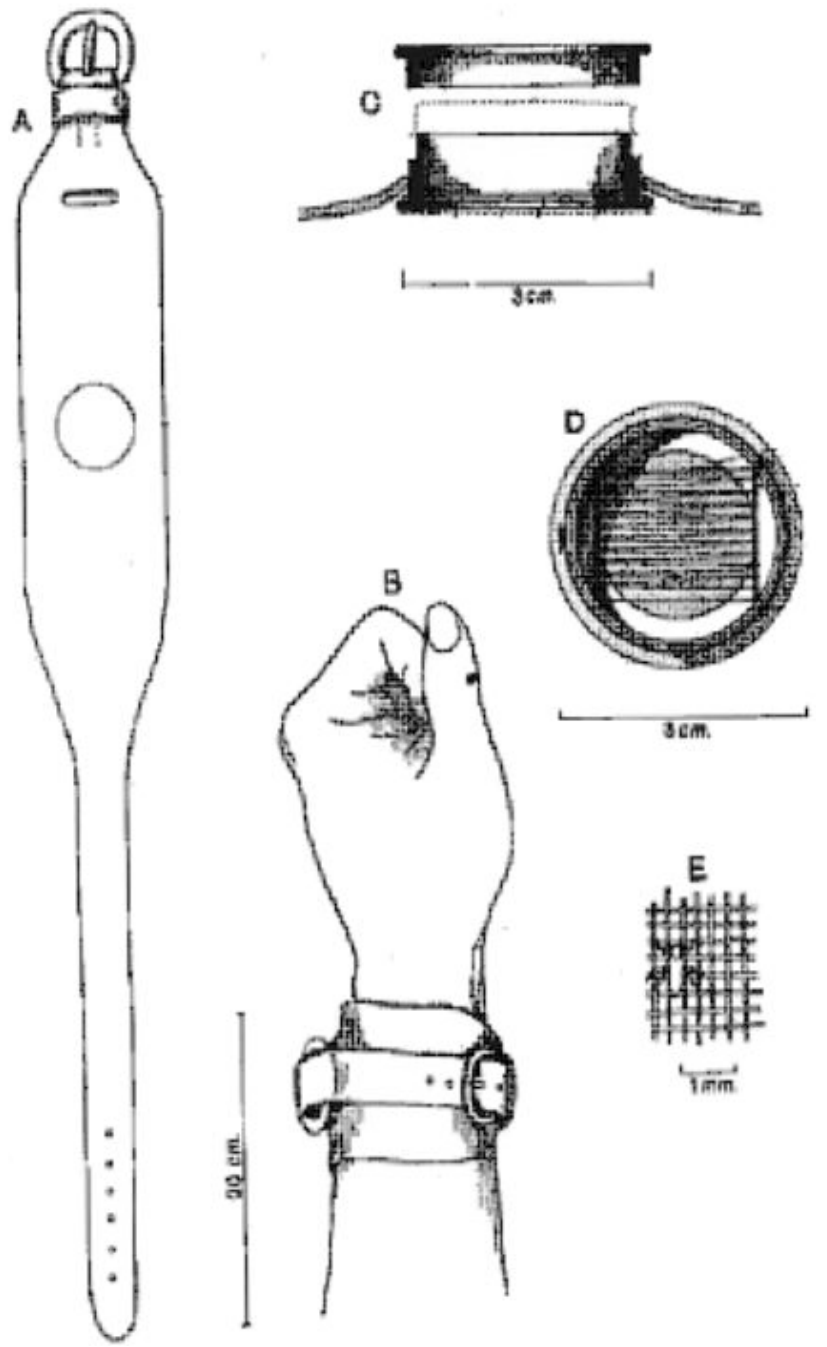

Fig. (1). The "wristlet method" for continuous feeding of head lice, as described by Nuttall in 1917 [14].

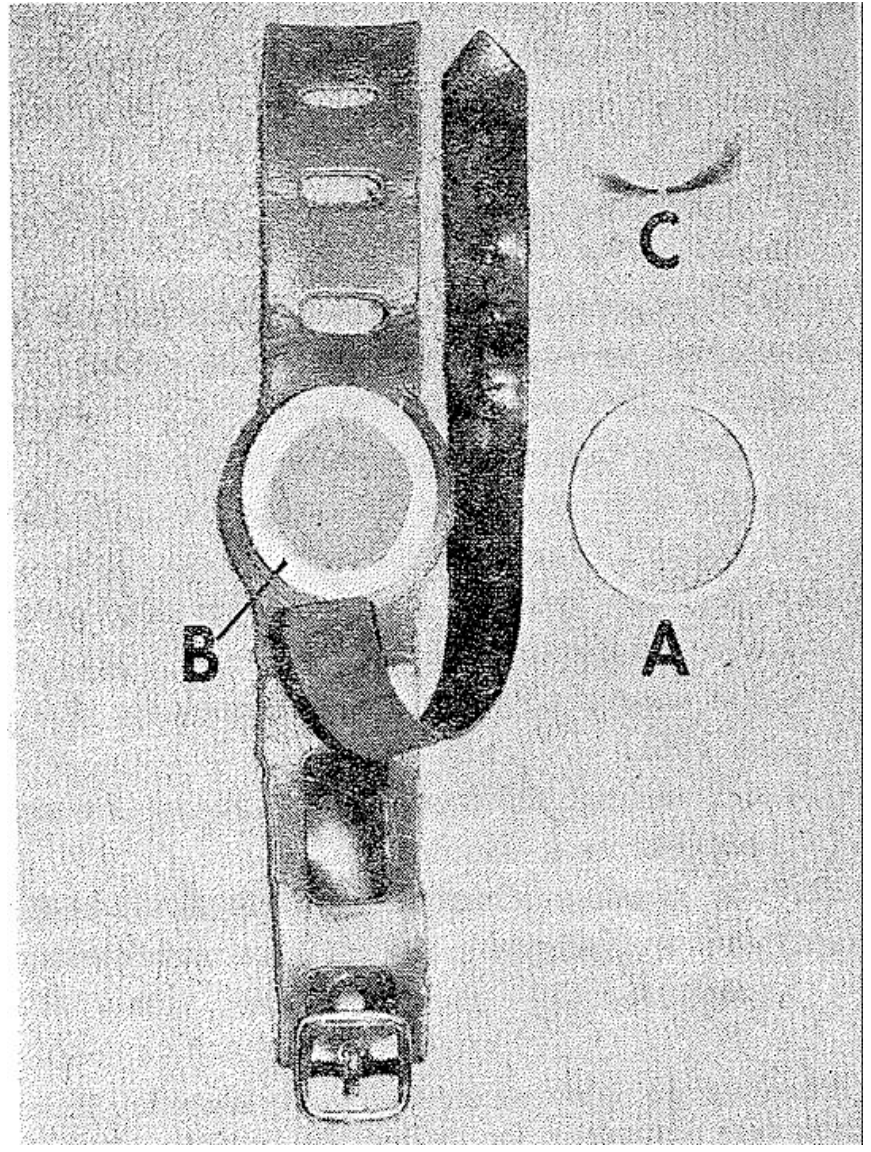

Fig. (2). Device for continuous feeding of head lice as used by Lang and Roan (1974) [15]. (A) Floor, (B) Roof, (C) Hair sample.

Here we present a further adaptation of these historical methods, optimizing egg production and ex vivo hatch rates. We describe development of eggs over time, and the age of head lice eggs is matched with the development of morphological characteristics.

\section{MATERIAL AND METHODS}

\section{Head Lice Used}

Adult head lice (Pediculus humanus capitis) were obtained by dry combing from children living in a resourcepoor community in the city of Fortaleza (northeast Brazil). In this community, head lice are hyperendemic [18]. Children had not been treated for head lice infestations nor received antiparasitic or antibiotic drugs during the previous four weeks. Combing was performed over a white sheet of paper, in order to easily recognize lice. A magnifying glass was used in the case of doubt.

\section{Head Lice Feeding}

Head lice were fed after collection, in order to obtain fertile eggs of known age. Two methods of feeding were applied: interval feeding and continuous feeding.

Interval feeding was performed on the back of the hand of the investigators, approximately every 8-12 hours (Figs. 3a and 3b). The lice, attached to strands of hair, would rapidly search the skin and begin blood meals. The feeding interval ended when lice stopped feeding and started to wander around on the skin (after 5-15 minutes). Between 
blood meals, head lice were incubated in Petri dishes on moistened filter paper with strands of hair. The Petri dish was cleaned every 24 hours, the hair strands with freshly laid eggs attached collected, and new hair strands were provided.

(a)

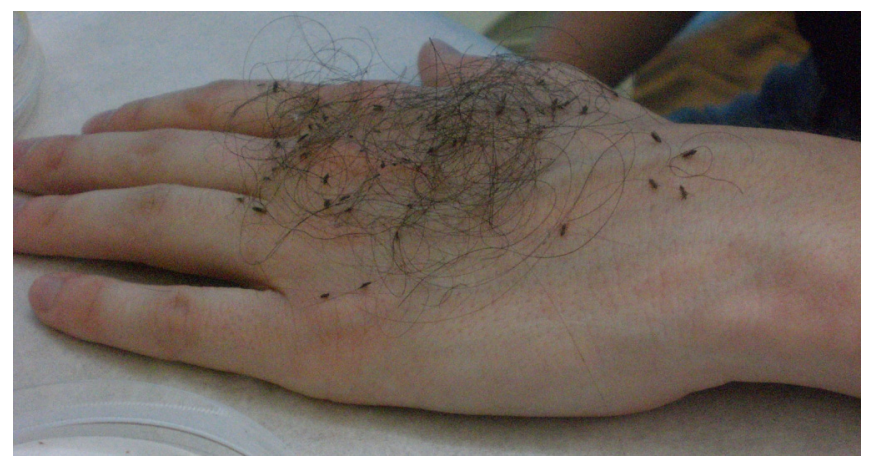

(b)

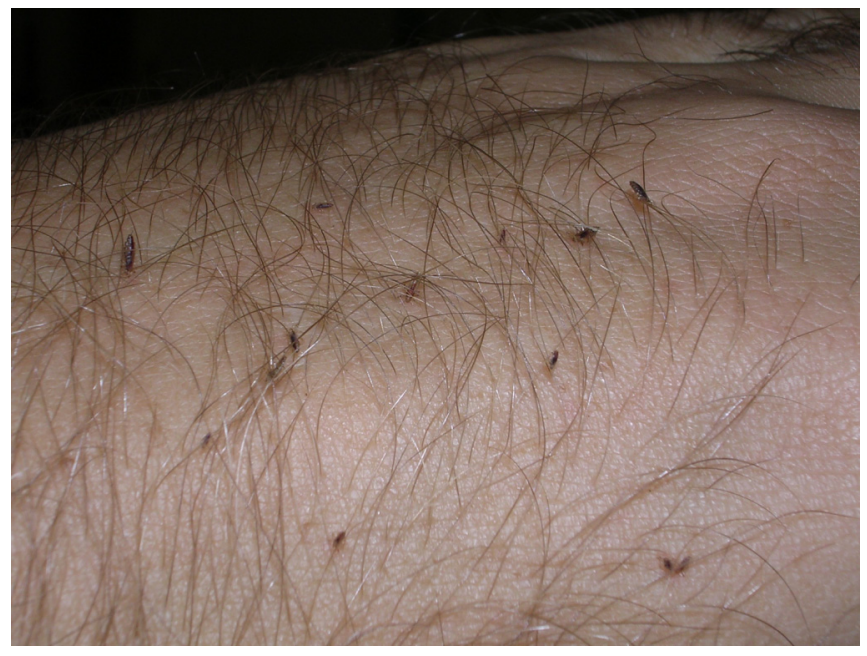

Fig. (3a, b). Interval feeding of head lice on the hands of the investigators.

Continuous feeding was based on descriptions by Nuttall (1917) [14], as adapted by Lang \& Roan (1974) [15], and Takano-Lee et al. (2003) [12]. We adapted further the method as shown in Fig. (4) different samples of hair were placed in a receptacle for oviposition. Head lice were placed in a round Plexiglas receptacle ("artifical dog", Flea Data Inc., Freeville, NY, USA), closed with gauze (Adaptic 2013 Non - Adhering -Dressing 3/7.6cm x $20.3 \mathrm{~cm}$, Johnson + Johnson Wound Management, washed with soap). The gross openings of the gauze would not prevent small lice from escaping, but facilitate handling when the device was positioned on the skin. The device was tightly secured to the skin by a gauze bandage. The receptacle was placed onto the skin of a volunteer for $24 \mathrm{~h}$ per day to simulate natural conditions (Fig. 4). Head lice would be exposed to a similar temperature as compared to living conditions on the scalp, and had continuous access to blood meals. The artificial dog was cleaned every 24 hours, the hair strands with eggs attached were collected, and new hair strands were provided. Continuous feeding was performed for 2-5 consecutive days with three short periods per day without feeding of approximately 15 minutes. During this period, volunteers (four of the authors) were advised to change the feeding sites twice daily, avoiding irritated areas caused by prior feeding. No oral medication e.g. antibiotics or ivermectin was used during the period of the study.

\section{Incubation of Eggs}

Incubation of head lice eggs, either obtained by interval feeding or continuous feeding, was performed within ideal conditions of temperature $\left(25-28,6^{\circ} \mathrm{C}\right)$ and relative humidity $(51,8 \%-69,3 \%)$, as described recently by Mougabure Cueto et al. [19]. Eggs were kept in Petri dishes on Whatman filter paper exposed to a $12 \mathrm{~h}$ day-night rhythm for a minimum of 14 days. Every three days, the head lice eggs were washed, to mimic the in vivo situation of hair washing: eggs, attached to hair strands, were placed into a water-shampoo solution containing no potentially pediculicidal compounds for one minute and then rinsed in tap water for 15 seconds.

\section{Outcome Measures}

The following outcome measures were used:

1. Signs of development: appearance of a so-called "eye spot" and presence of embryonic structures.

2. Signs of viability: embryonic movements - pulsating air bubble at the rear of the egg or limb movement visible during a $15 \mathrm{sec}$ examination period under a dissecting microscope.

3. Hatching: hatching was defined as the presence of an open operculum and an empty egg shell.

Eggs were observed daily regarding the outcome measures using a dissection microscope, for a period of 14 days after oviposition. Eggs obtained by intermittent feeding $(n=159)$ were only observed once after 14 days, according to the hatching status. A total of 340 eggs were obtained by continuous feeding. Of these, 205 were examined daily in batches of 10, according to all three outcome measures, and the remaining 135 only once after 14 days, to confirm hatching status.

To calculate the rate of development, the number of eggs with presence of an eye spot/embryonal structures and the number of hatched eggs were added and divided by the total number of eggs observed $(\mathrm{n}=205)$.

In addition, 10 eggs obtained by continuous feeding were photographed daily under a dissecting microscope, in order to document embryonic development visually.

Examinations were done by a single observer (S.S.) to prevent inter-observer variation. Data were entered using Excel spreadsheets and checked for entry errors. Binomial 95\% confidence intervals were calculated using STATA, version 10 (Stata Corporation, College Station, USA). Chi square test was used to compare relative frequencies.

\section{RESULTS}

\section{Development of Eggs and Signs of Viability}

Embryonal eye spots started to appear 6 days after oviposition, and darkened from light red to black. Embryonic structures became more apparent with time, so that limbs could be distinguished within the egg (Fig. 5). Median time until the appearance of an eye spot was 7 days (interquartile range 6,5 - 8 days). Embryonic structures and eye spots 

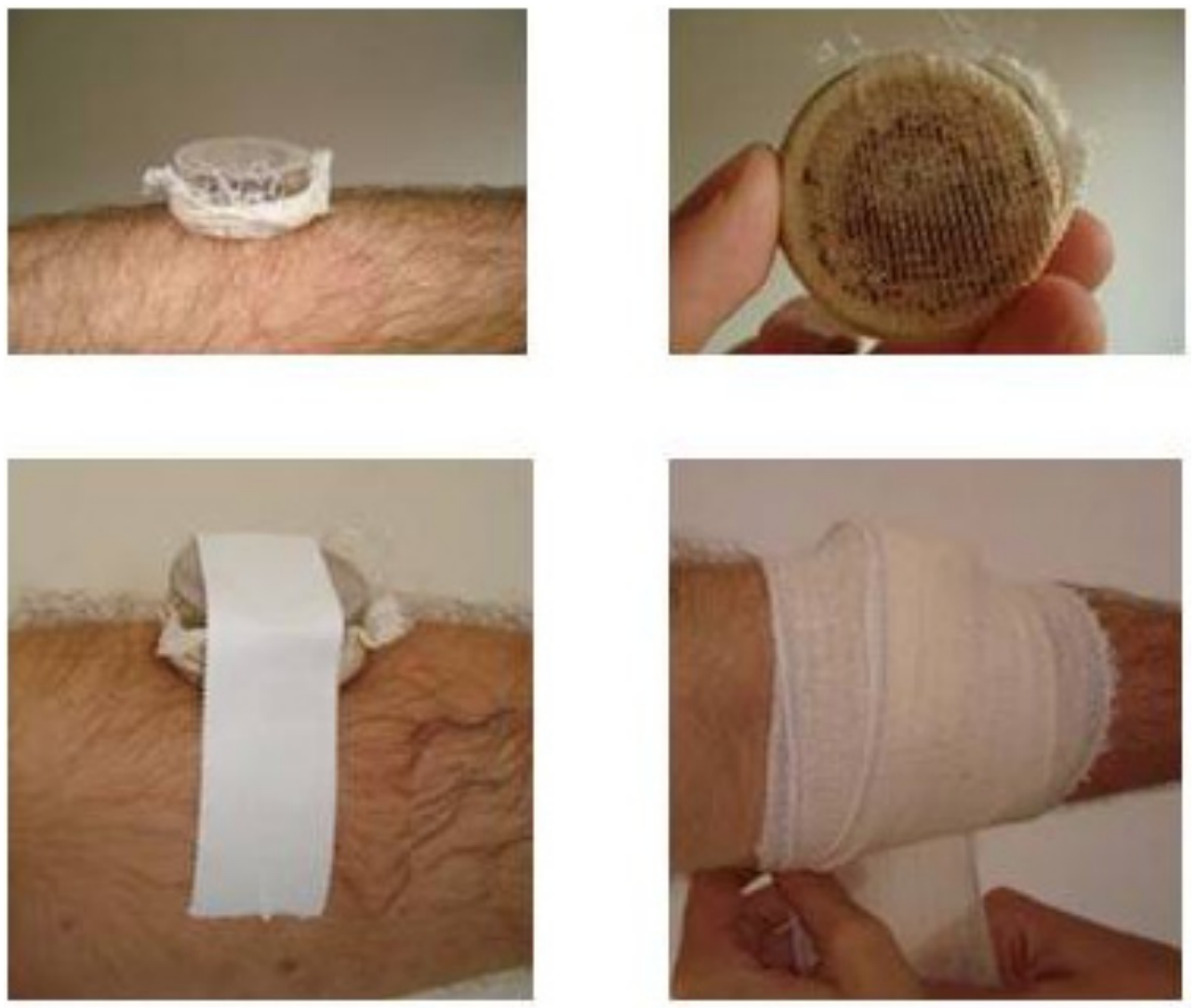

Fig. (4). Continuous feeding on the forearm using an "artificial dog".

appeared simultaneously. The fraction of developed eggs over time is detailed in Fig. (6).

Embryonic movements were first seen 9 days after oviposition (median $=11$ days; interquartile range: 10-11
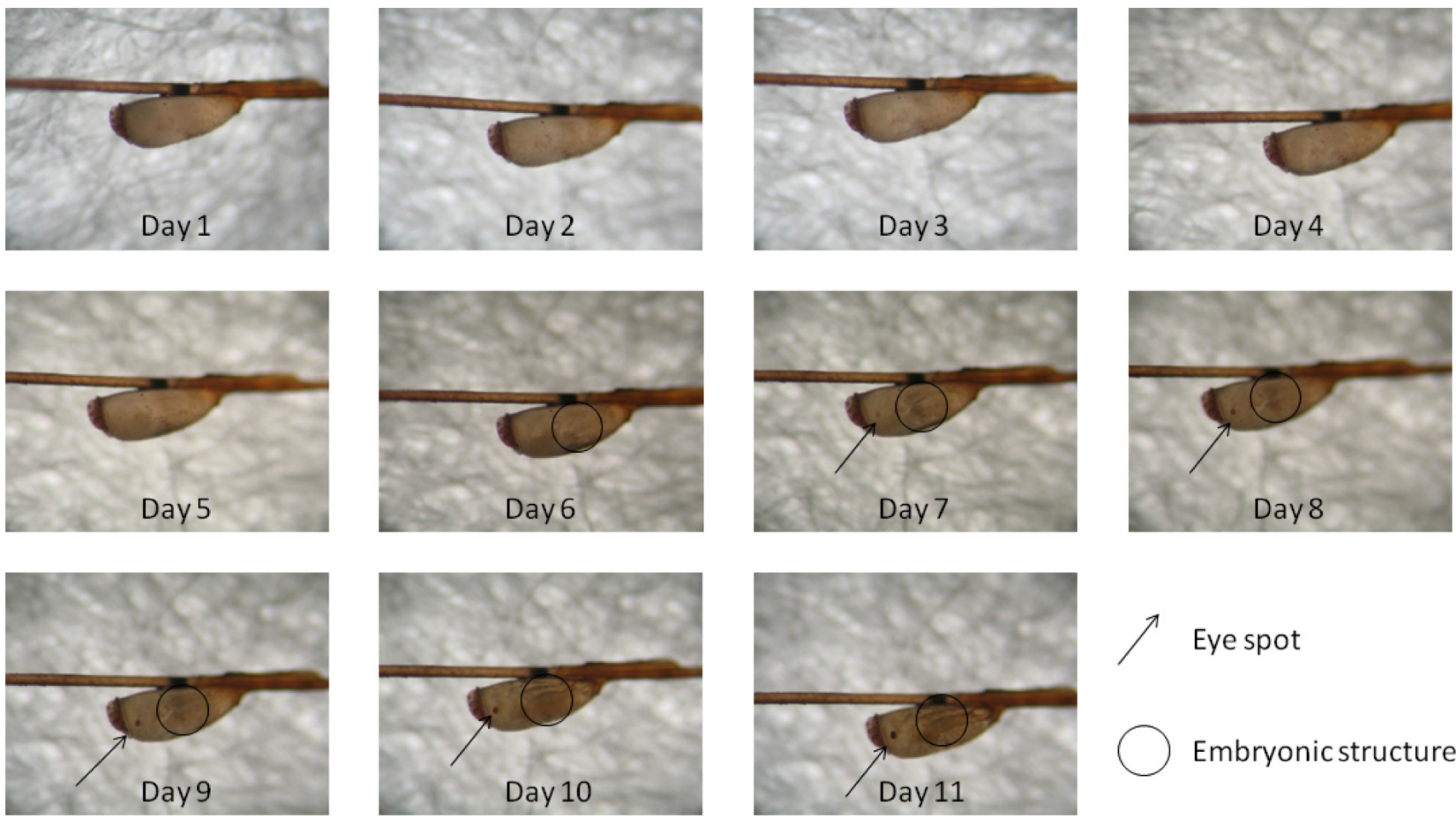

days). In about $60 \%$ of the 205 eggs embryonic movements were observed on days ten and eleven after oviposition (Fig. 7).
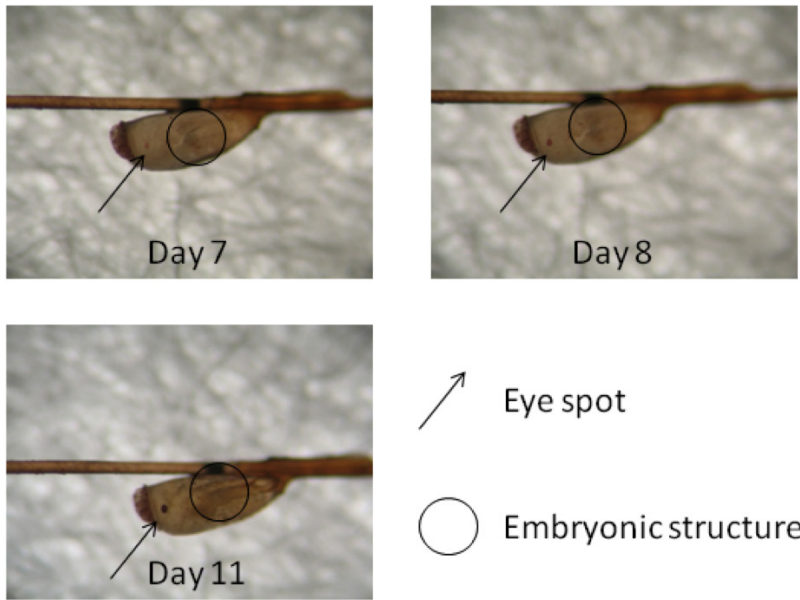

Eye spot

Embryonic structures

Fig. (5). Development of a head louse egg over time. 


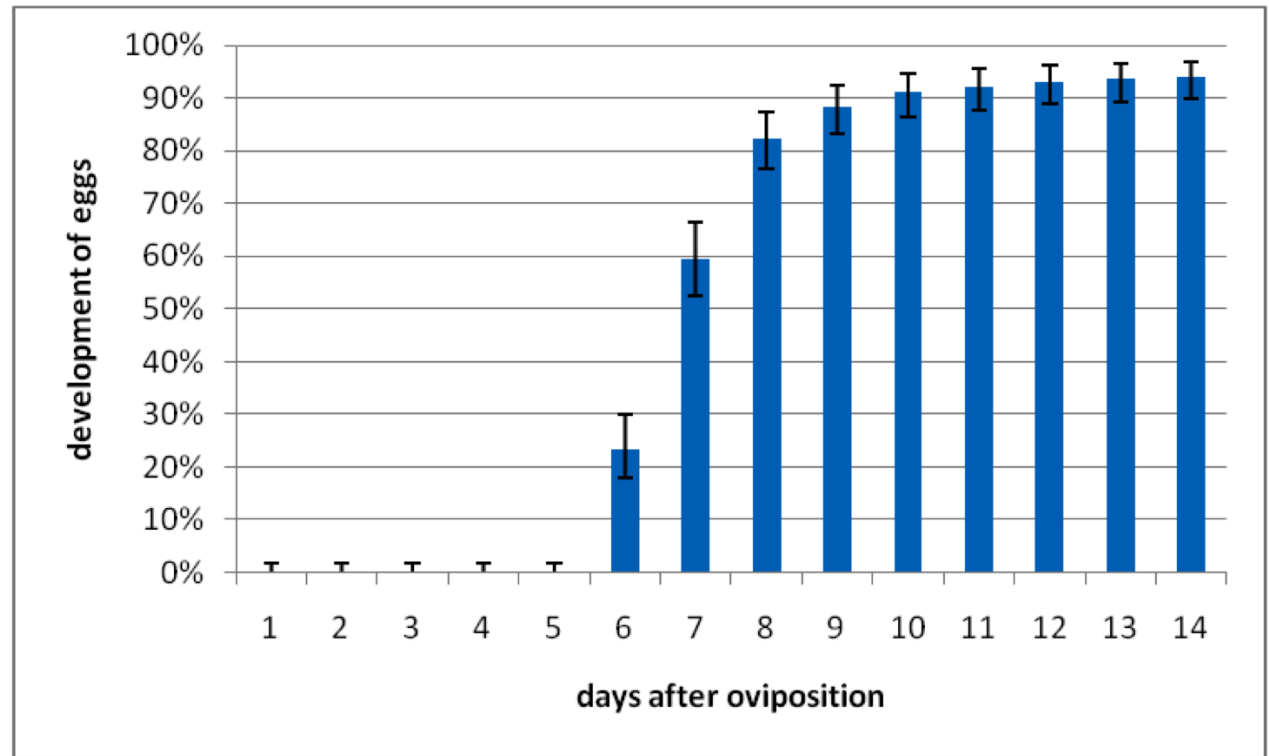

Fig. (6). Fraction of developed eggs (presence of eye spots/embryonic structures, hatched eggs) over time ( $\mathrm{n}=205$, continuous feeding). Vertical bars indicate $95 \%$ confidence intervals.

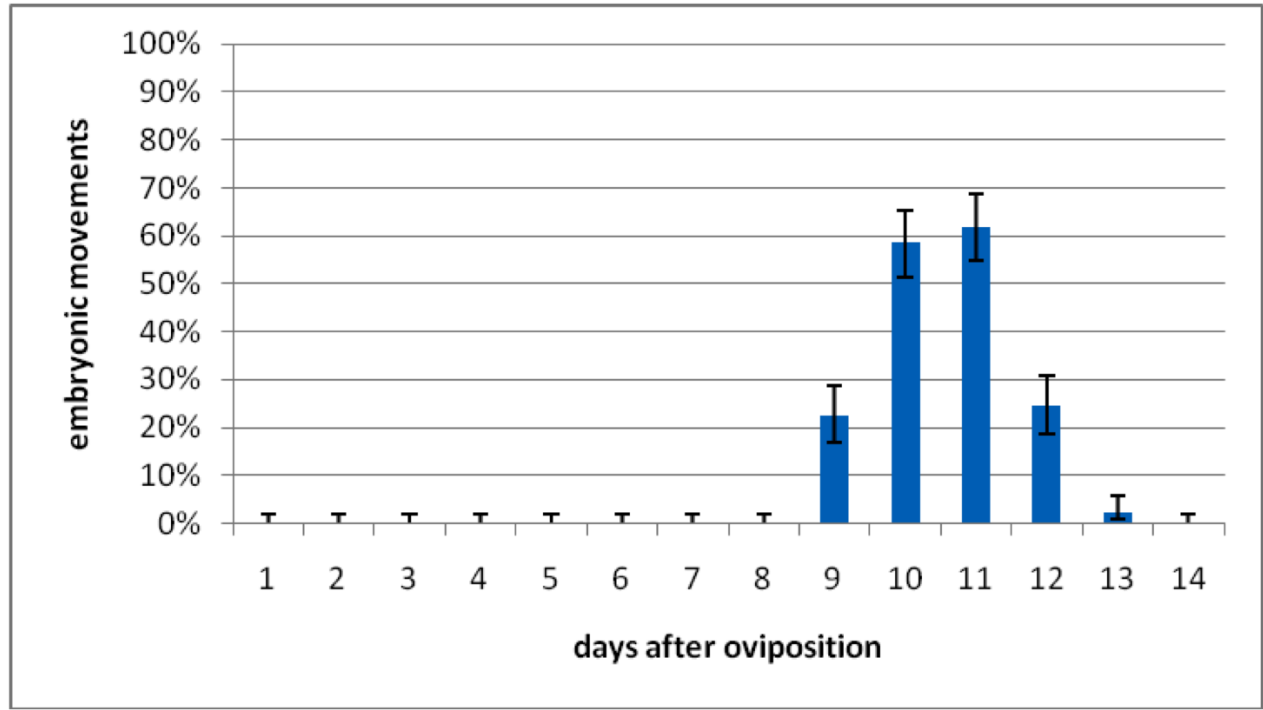

Fig. (7). Fraction of head lice eggs with visible embryonic movements ( $\mathrm{n}=205$, continuous feeding of adult lice). The reduced values on day 12 and later are caused by the increased number of hatched eggs. Vertical bars indicate $95 \%$ confidence intervals.

\section{Hatch Rates}

Interval feeding of head lice revealed a hatch rate of $10,7 \%(17 / 159)$, as compared to $80,9 \%(275 / 340)$ achieved with continuous feeding $(\mathrm{p}<0,0001)$. All eggs hatched within a period of 14 days. No hatching occurred after this period.

Hatch rates after continuous feeding of adult lice are shown in Fig. (8). Of the 205 eggs observed daily, 167 $(81,5 \%)$ hatched. Hatching started 9 days after oviposition (median time to hatching $=12$ days, interquartile range: 11 13 days). Fig. (9) shows the hatching of a nymph.

Fig. (10) summarizes median values and dispersion of the outcome measures. It is apparent that $95 \%$ of eggs developed eye spots until the $10^{\text {th }}$ day and that $90 \%$ of eggs hatched between the $11^{\text {th }}$ and $13^{\text {th }}$ day.

\section{Skin Irritation}

Volunteers experienced little skin irritation when first feeding head lice. After a period of sensitization against components of head lice saliva, skin irritation in the form of a papular rash and moderate to severe itching appeared (Fig. 11). Irritation increased with the number of lice fed and the time of feeding. After application of topical corticosteroids, itching was reduced considerably. Symptoms continued for a maximum of seven days and disappeared without any complications. Excoriations due to scratching were observed.

\section{DISCUSSION}

Standard procedures for testing ovicidal properties of pediculicides are needed for a reliable evaluation of products marketed against head lice infestations [8]. Burkhart et al. 


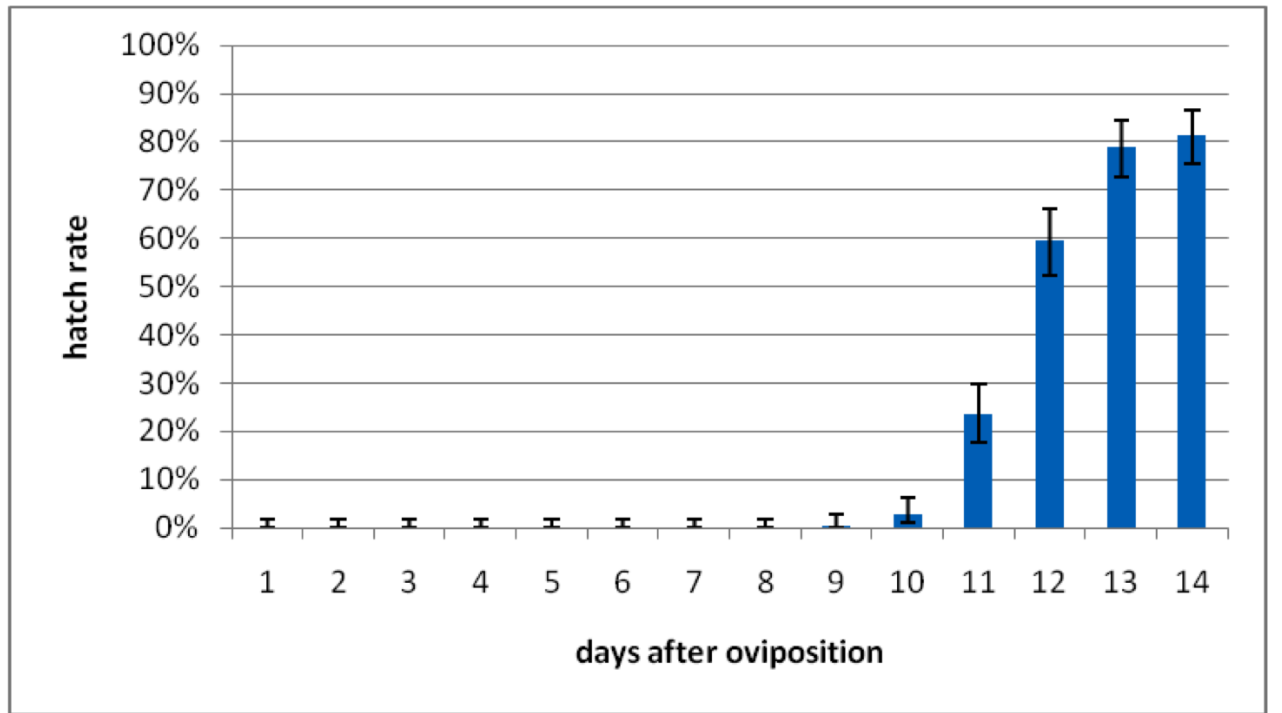

Fig. (8). Hatch rate of head lice eggs over time ( $\mathrm{n}=205$, continuous feeding of adult lice). Vertical bars indicate $95 \%$ confidence intervals.
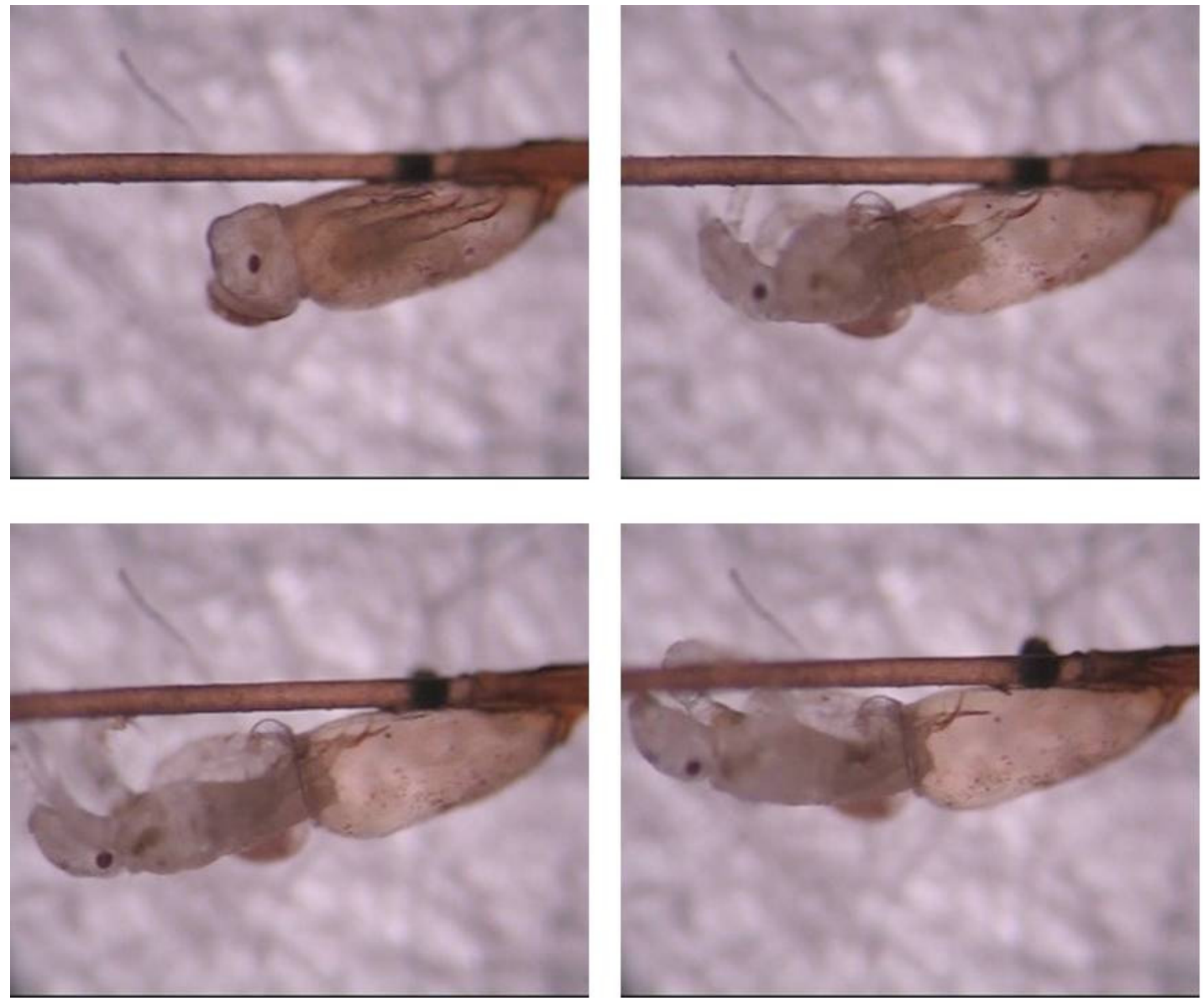

Fig. (9). Hatching of a head louse.

[10] proposed standard procedures, but relied on eggs obtained from infested individuals. Comparative testing, though, is more reliable when eggs are standardized in age or development obtained under controlled laboratory conditions.

We achieved a high hatch rate after applying continuous feeding of head lice on volunteers. Head lice eggs obtained from continuous feeding on a human host are exposed to

conditions similar to their natural habitat. Similar methods have been applied previously by Takano-Lee et al. [11] (76\% hatch rate) and Yang et al. [20] (83\% hatch rate). The high hatch rates underline the suitability of this rearing method. The significantly lower hatch rates obtained after intermittent feeding of head lice adults may reflect the need of head lice to feed repeated times in order to mate and lay eggs. In addition, there is the advantage of known age and 


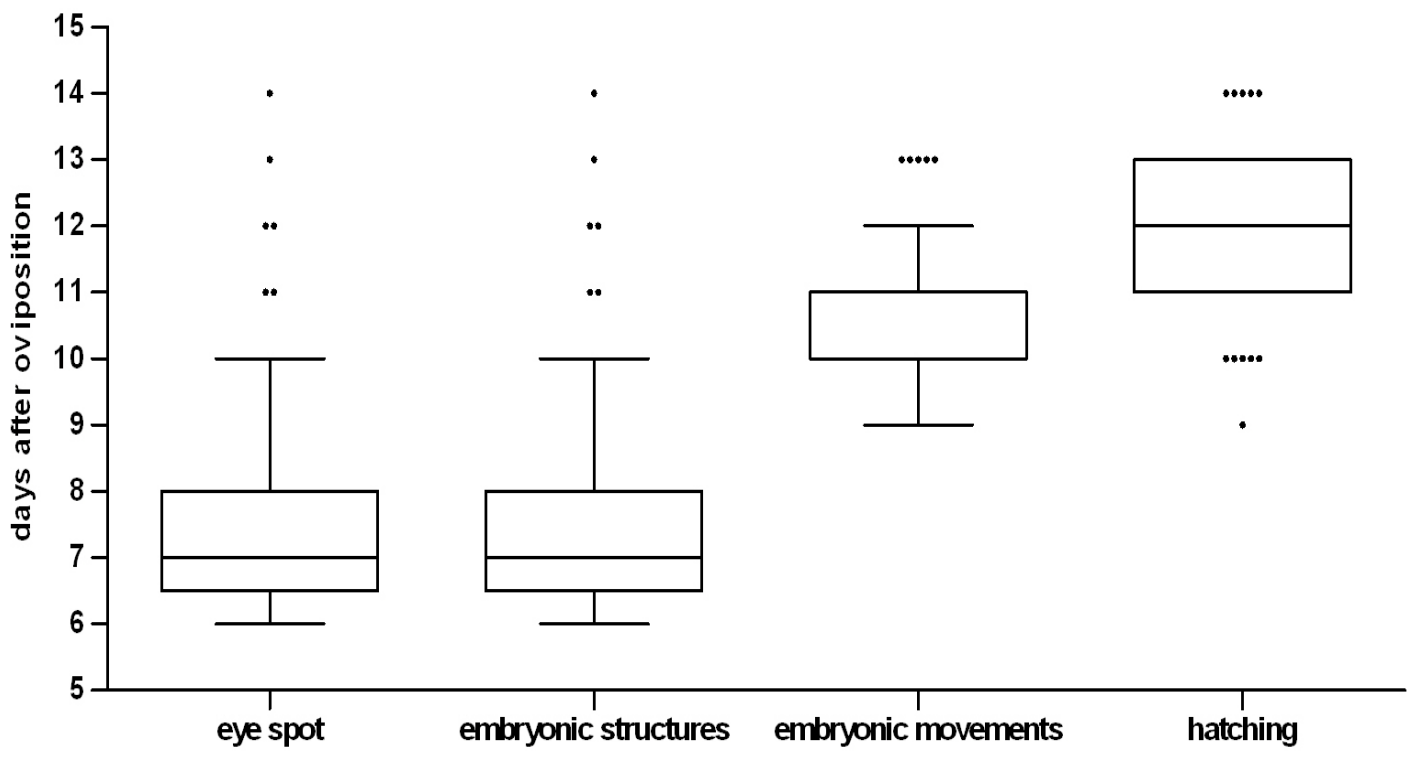

Fig. (10). Overview of the time of appearance of different outcome measures. Horizontal lines indicate the median values; the ends of the boxes the upper (75\%) and lower (25\%) quartiles; the whiskers the $5 \%$ and $95 \%$ percentiles; and data points outside the fences outlier values $(\mathrm{n}=205$ eggs, continuous feeding of adult lice).

therefore the possibility to evaluate differences in pediculicide susceptibility according to the age of eggs [9]. The continuous feeding method is simple to establish and cheap to conduct. We have previously used this method and have convincingly shown that high concentration dimeticone products have a high ovicidal efficacy, as compared to low concentration dimeticone, permethrin and pyrethrum [9].

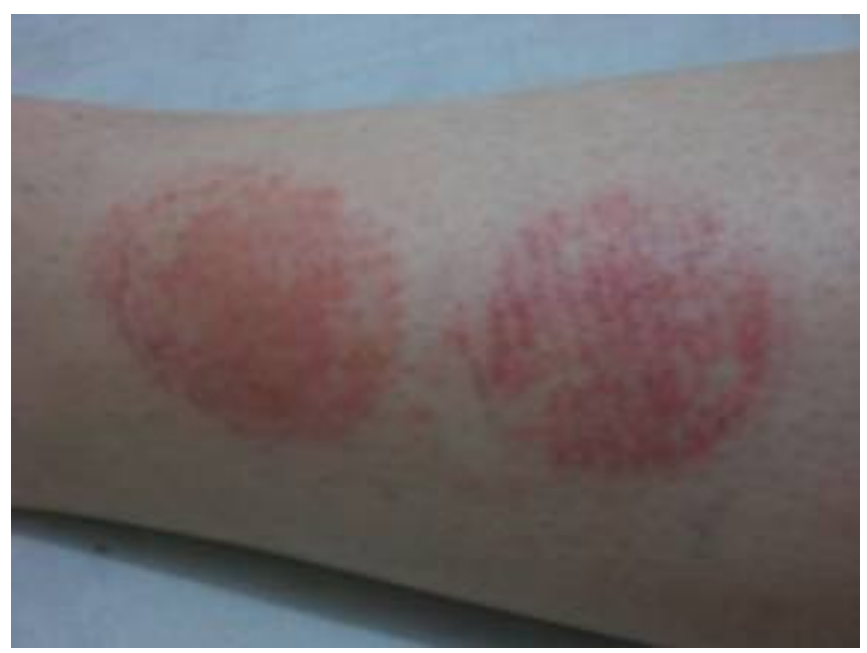

Fig. (11). Skin irritation after a 12-hour feeding period on the lower leg of a volunteer.

However, irritation caused by feeding head lice on volunteers is arduous, and as a consequence, alternative methods have been proposed. First, testing of ovicidal efficacy of pediculicides can be conducted on head lice eggs collected from infested individuals. Hatch rates using this approach range between $90 \%$ and $95 \%$ [21-25] but there are only indirect markers to determine the eggs' age, such as their color [19]. Therefore, pediculicide testing according to age or developmental stage is limited. Another approach is the use of laboratory based colonies of body lice [26, 27].
This approach includes another subspecies of lice, which differs in life cycle, habitat and feeding behavior from head lice, but arguably physiological characteristics are similar. Body lice are easily adapted to the laboratory environment. For greater convenience, they were trained to feed on rabbits in predefined intervals instead of feeding on humans continuously. Clearly, a laboratory colony of body lice cannot mimic the current pattern of resistance against pediculicides. Thus, results from ovicidal efficacy testing on colony-derived body lice eggs should be treated with great caution.

A third possibility for ovicidal testing is the use of head lice eggs after rearing lice in vitro, as developed and applied by Takano-Lee et al. $[11,12]$. Head lice were fed through a Parafilm membrane on human blood and reared in an automated feeding apparatus. Hatch rates of eggs were only $38 \%$, which limits their use in ovicidal assays. The authors observed by in vivo rearing of head lice $(76,2 \%)$, and a manual in vitro rearing system (78\%) significantly higher hatch rates. High concentrations of antibiotics added to the blood in the automated feeding apparatus might have negatively affected the fecundity of head lice eggs [11]. Antibiotics have been discussed as a treatment option for pediculosis, as they may eliminate symbiotic bacteria found in the lices' guts [2, 28-30]. Unfortunately, only first instar nymphs can be trained to feed through the membrane, so that an a priori selection of lice occurs, resulting in a strain of lice distinct from wild strains, especially when maintained in the apparatus for various generations. Consequently, low hatch rates of eggs, the need for extensive antibiotic treatment of the blood supply in the automated apparatus and selected strains of head lice living in the apparatus limit the interpretation of results obtained of ovicidal testing using eggs reared in the apparatus.

By using the continuous feeding method, we were able to describe in a detailed way the development of head lice eggs 
according to age under controlled laboratory ex vivo conditions. We matched external features of development to the age of head lice eggs. Mougabure Cueto et al. [19] used developmental criteria to classify head lice eggs into three developmental stages; early, medium and late embryos. The features described by Mougabure Cueto et al. were also observed in our experiment, but age of head lice eggs in that study was not known, because the eggs had been collected from infested individuals.

Lebwohl (2007) [29] described the life cycle of head lice and states that hatching occurred after 7-12 days, while our findings showed a delay of two days, with hatching starting on day 9. The delay in our experiments might be due to the lower temperature of incubation, as compared to the human scalp, known to delay development and hatching [14].

Features of embryonic development, as the appearance of an eye spot and embryonic structures may be used as additional outcome measures in ovicidal assays. The time of appearance noted in our experiments can serve as an indicator for normal embryonic development, and delay or inhibition in embryonic development can serve as an indicator for ovicidal action.

\section{CONCLUSION}

We describe in detail the development of head lice eggs ex vivo. The data are important for planning and development of ovicidal assays. Continuous feeding of adult lice on a human host is a suitable approach to obtain fertile head lice eggs of known age. The method is simple and cheap, offers conditions similar to the natural habitat, and hatch rates are high. However, side effects on volunteers are considerable.

\section{REFERENCES}

[1] Falagas ME, Matthaiou DK, Rafailidis PI, Panos G, Pappas G. Worldwide prevalence of head lice. Emerg Infect Dis 2008; 14: 1493-4.

[2] Burgess IF. Human lice and their control. Annu Rev Entomol 2004; 49: 457-81.

[3] Kristensen M, Knorr M, Rasmussen AM, Jespersen JB. Survey of permethrin and malathion resistance in human head lice populations from Denmark. J Med Entomol 2006; 43: 533-8.

[4] Vassena CV, Mougabure Cueto G, González Audino P, Alzogaray RA, Zerba EN, Picollo MI. Prevalence and levels of permethrin resistance in Pediculus humanus capitis De Geer (Anoplura: Pediculidae) from Buenos Aires, Argentina. J Med Entomol 2003; 40: 447-50.

[5] Durand R, Millard B, Bouges-Michel C, Bruel C, Bouvresse S, Izri A. Detection of pyrethroid resistance gene in head lice in schoolchildren from Bobigny, France. J Med Entomol 2007; 44: 796-8.

[6] Kasai S, Ishii N, Natsuaki M, et al. Prevalence of kdr-like mutations associated with pyrethroid resistance in human head louse populations in Japan. J Med Entomol 2009; 46: 77-82.

[7] Yoon KS, Gao JR, Lee SH, Clark JM, Brown L, Taplin D. PermethrinResistant Human Head Lice, Pediculus capitis, and Their Treatment. Arch Dermatol 2003; 139: 994-1000.

[8] Heukelbach J, Canyon DV, Oliveira FA, Muller R, Speare R. In vitro efficacy of over-the-counter botanical pediculicides against the head louse Pediculus humanus var capitis based on a stringent standard for mortality assessment. Med Vet Entomol 2008; 22: 264-72.

[9] Heukelbach J, Sonnberg S, Mello I, Speare R, Oliveira FA. High ovicidal efficacy of a dimeticone pediculicide: a new era of head lice treatment. J Am Acad Dermatol 2010; in press.

[10] Burkhart CN, Burkhart CG. Recommendation to standardize pediculicidal and ovicidal testing for head lice (Anoplura: Pediculidae). J Med Entomol 2001; 38: 127-9.

[11] Takano-Lee M, Velten RK, Edman JD, Mullens BA, Clark JM. An automated feeding apparatus for in vitro maintenance of the human head louse, Pediculus capitis (Anoplura: Pediculidae). J Med Entomol 2003; 40: 795-9.

[12] Takano-Lee M, Yoon KS, Edman JD, Mullens BA, Clark JM. In vivo and in vitro rearing of Pediculus humanus capitis (Anoplura: Pediculidae). J Med Entomol 2003; 40: 628-35.

[13] Smith CN, Eddy GW. Techniques for rearing and handling body lice, oriental rat fleas, and cat fleas. Bull World Health Organ 1954; 10: 12737.

[14] Nuttall GHF. The biology of Pediculus humanus. Parasitology 1917; 10: $180-4$.

[15] Lang JD, Roan CC. An improved method for rearing head lice. J Med Entomol 1974; 11: 112.

[16] Buxton PA. The louse: an account of the lice which infest man, their medical importance and control. $2^{\text {nd }}$ ed. London: Edward-Arnold Co. 1947.

[17] Peacock AD. The structure of the mouthparts and mechanism of feeding in Pediculus humanus. Parasitology 1919; 11: 98-117.

[18] Heukelbach J, van Haeff E, Rump B, Wilcke T, Sabóia Moura RC, Feldmeier H. Parasitic skin diseases: health care-seeking in a slum in north-east Brazil. Trop Med Int Health 2003; 8: 368-73.

[19] Mougabure Cueto GA, Zerba EN, Picollo MI. Embryonic development of human lice: rearing conditions and susceptibility to spinosad. Mem Inst Oswaldo Cruz 2006; 101: 257-61.

[20] Yang YC, Lee SH, Clark JM, Ahn YJ. Ovicidal and adulticidal activities of Origanum majorana essential oil constituents against insecticide-susceptible and pyrethroid/malathion-resistant Pediculus humanus capitis (Anoplura: Pediculidae). J Agric Food Chem 2009; 57: 2282-7.

[21] Carpinella MC, Miranda M, Almirón WR, Ferrayoli CG, Almeida FL, Palacios SM. In vitro pediculicidal and ovicidal activity of an extract and oil from fruits of Melia azedarach L. J Am Acad Dermatol 2007; 56: 250-6.

[22] Gomez Urcuyo F, Zaias N. Malathion lotion as an insecticide and ovicide in head louse infestation. Int J Dermatol 1986; 25: 60-2.

[23] Anbu Jeba Sunilson JS, Radhamani S, Gopinath R, Kalusalingam A, Vimala AG, Husain HA. In vitro screening of anti-lice activity of Pongamia pinnata Leaves. Korean J Parasitol 2009; 47: 377-80.

[24] Toloza AC, Zygadlo J, Mougabure-Cueto G, Zerba E, Faillaci S, Picollo MI. The fumigant and repellent activity of aliphatic lactones against Pediculus humanus capitis (Anoplura: Pediculidae). Mem Inst Oswaldo Cruz 2006; 101: 55-6.

[25] Maunder JW. Clinical and laboratory trials employing carbaryl against the human head-louse, Pediculus humanus capitis (de Geer). Clin Exp Dermatol 1981; 6: 605-12.

[26] Veal L. The potential effectiveness of essential oils as a treatment for headlice, Pediculus humanus capitis. Complement Ther Nurs Midwifery 1996; 2: 97-101.

[27] Priestley CM, Burgess IF, Williamson EM. Lethality of essential oil constituents towards the human louse, Pediculus humanus, and its eggs. Fitoterapia 2006; 77: 303-9.

[28] Meinking TL. Infestations. Curr Probl Dermatol 1999; 11: 77-114.

[29] Lebwohl M, Clark L, Levitt J. Therapy for head lice based on life cycle, resistance, and safety considerations. Pediatrics 2007; 119: 965-74.

[30] Aschner M. Studies on the symbiosis of the body louse. 1. Elimination of the symbionts by centrifugation of the eggs. Parasitology 1934; 26: $309-14$. 